\title{
Alcoholic Brain Atrophy
}

National Cancer Institute

\section{Source}

National Cancer Institute. Alcoholic Brain Atrophy. NCI Thesaurus. Code C97043.

Atrophy of the cerebral cortex and/or cerebellum due to chronic ethanol consumption. 